\title{
Air-coupled Ultrasonic Inspection with Adaptive Lamb Wave Control
}

\author{
Manfred Schönheits@a ${ }^{\mathrm{a}}$, Armin Huber $\bigodot^{\mathrm{b}}$ and Philipp Gänswürger \\ German Aerospace Center (DLR), Institute of Structures and Design, Augsburg, Germany \\ \{manfred.schoenheits, armin.huber, philipp.gaenswuerger $\} @ d l r . d e$
}

Keywords: $\quad$ Non-destructive Testing, Ultrasonic Inspection, Lamb Wave, Automation, Robotics.

\begin{abstract}
Single-sided air-coupled ultrasonic inspection has some beneficial properties compared to water-coupled ultrasonic inspection or double-sided ultrasonic testing. The absence of the need for water leads to easier process handling on the one hand e.g. when manufacturing aircraft components. On the other hand, because the process is single-sided, reachability is a minor problem compared to double-sided testing and end-effectors and fixtures can be designed in a less complex and more compact way. However, the nature of lamb waves requires that the geometrical relation of the transmitter and the receiver varies during the inspection process. In this paper, a prototype of an adaptive end-effector is introduced that was developed to implement this requirement and results of first evaluation tests are presented.
\end{abstract}

\section{INTRODUCTION}

Aerospace vehicles are constructed from an increasing amount of carbon composites because their strength-to-weight ratio compared to traditional metallic components can yield weight savings that enable these vehicles to operate more efficiently. However, the manufacturing of composites is still very expensive due to the high amount of manual labor that is involved and the use of high-performance but also high-cost prepreg material. Therefore, it is the aim of the Center for Lightweight Production Technology (ZLP) of the German Aerospace Center (DLR) to develop automated manufacturing process technology for large-scale components made from carbon fiber reinforced plastics (CFRP).

In order to guarantee cost-efficiency, processintegrated quality assurance based on non-destructive inspection (NDI) methods is essential. It has been concluded that air-coupled ultrasonic testing (ACUT) is a suitable NDI method (Ullmann et al., 2012). A NDI method has to meet certain key criteria: Fast measurement speed and data evaluation as well as the ability to inspect large components. This can only be achieved through automation of the inspection process.

The single-sided ACUT mode, which we have utilized in this work, is based on the excitation of Lamb waves in a component to be tested. This mode is

\footnotetext{
a (iD) https://orcid.org/0000-0002-5646-2111

b (i) https://orcid.org/0000-0002-5694-8293
}

also referred to as Focused Slanted Reflection Mode (FSRM). Lamb waves have been used for NDI purposes for many decades. An early description of the flaw detection of sheets and tubes immersed in water by means of Rayleigh and Lamb waves was given by Viktorov already back in 1967 (Viktorov, 1967). Only a few years later, Luukkala et al. proposed a contactless test method for paper and metal plates based on Lamb waves (Luukkala et al., 1971; Luukkala and Meriläinen, 1973). Many applications have been established since then, and the advent of composite materials in automotive and aerospace industries, which took place in the early 1990s, has added significant complexity to the non-destructive testing and evaluation processes. The ability of guided waves to propagate many meters in a waveguide is utilized for pipe inspection (Wilcox et al., 2001; Lowe et al., 1998). They are also used for the inspection of bonding (Lowe and Cawley, 1994), which is one of the most challenging tasks, especially in the case of kissing bonds (Kundu et al., 1998). Other relevant studies concerning NDI and structural health monitoring (SHM) on composite structures are found in Refs. (Maslov and Kundu, 1997; Kessler et al., 2002; Toyama et al., 2003; Su et al., 2006; Diamanti and Soutis, 2010; Purekar and Pines, 2010; Ramadas et al., 2011; Cunfu et al., 2013). The air-coupled version of the guided wave inspection could play an important role in future production lines. Often, the presence of a liquid coupling medium is unwanted because it might inflict damage to unsealed composite 
structures. All of the following researchers have used the air-coupled variant. Castaings et al. have done significant work on the single-sided ultrasonic testing of composites by using Lamb waves (Castaings and Cawley, 1996; Castaings et al., 1998; Castaings and Hosten, 2001; Castaings and Hosten, 2008), while Solodov et al. have used them for transmissive inspection (Solodov et al., 2004b; Solodov et al., 2004a; Solodov et al., 2006).

In order to excite Lamb waves, the ultrasound transducers must follow a certain orientation with respect to the specimen which we call excitation angle. This excitation angle depends upon certain properties of the component, which might change from one location to another. Therefore, the inspection process has to be interrupted and the orientation of the ultrasonic transducers must be changed such that the correct excitation angle is met. In this paper, we present a prototype of a fully automated, adaptive end-effector (AEE), which enables the continuous adjustment of the ultrasonic transducers such that Lamb waves are excited at optimal efficiency at any location on the component.

\section{PROBLEM DESCRIPTION}

A Lamb wave propagating through a plate causes displacement throughout its whole thickness. Since ultrasound is reemitted along the propagation path of the Lamb wave, it can be detected with the receiver. As depicted in figure 1, both sender and receiver must be oriented at the same angle with respect to the surface normal when used on a flat specimen. A flaw causes a change in the displacement amplitude, and can be detected thereby. A beam shield made of cardboard is mounted between the two transducers to prevent sound propagating directly from the sender to the receiver. Both transducers can be rotated and moved horizontally and vertically, but only manually. The industrial robot scans the specimen while ultrasonic pulses are triggered such that pairs of 6DOF measurement points and the corresponding ultrasonic data are generated. By that way, three-dimensional ultrasonic images of a specimen can be obtained and flaws localized. However, because the transducers are fixed, this solution is limited to parts with constant thickness and zero or constant curvature.

According to Snell's law, the excitation angle $\theta$ with respect to the surface normal is given by

$$
\theta=\sin ^{-1}\left(\frac{v_{\mathrm{I}}}{v_{\text {Lamb }}}\right),
$$

where $v_{\mathrm{I}}$ is the phase velocity of the incident plane wave and $v_{\text {Lamb }}$ the phase velocities of the Lamb wave

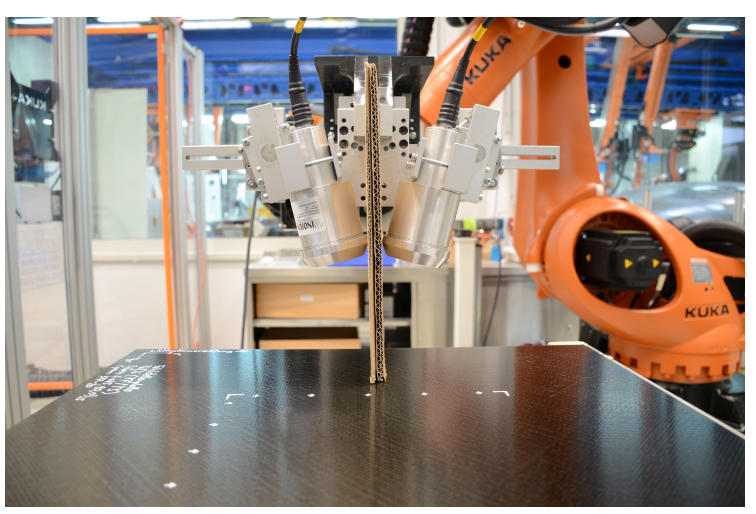

Figure 1: Conventional end-effector for air-coupled ultrasonic inspection by using Lamb waves.

excited in the plate (Castaings and Cawley, 1996). While $v_{\text {I }}$ is known, e.g., $343 \mathrm{~m} / \mathrm{s}$ at room temperature in air, $v_{\text {Lamb }}$ is not. $v_{\text {Lamb }}$ depends upon

- The stiffness of the specimen's material,

- The layup (fiber orientation and layer thickness) of the specimen,

- The ultrasonic transducer frequency since Lamb waves are dispersive.

With the known stiffness matrix and the layup, one can calculate Lamb wave dispersion diagrams ( $\theta$ vs. frequency) for a given propagation direction in the laminate. A discussion of this procedure is beyond the scope of this paper. In our previous work, we have created an interactive software called Dispersion Calculator (DC) with MATLAB ${ }^{\circledR}$ (MathWorks, Natick, MA, USA) for the calculation of Lamb wave dispersion diagrams (Huber and Sause, 2018). The standalone software can be downloaded free of charge on the DLR-homepage ${ }^{1}$.

Real structural parts generally do not have a constant thickness or a homogeneous layup. For an automated inspection process this means that the necessary poses of the transducers are not constant but depend on the location. Figure 2 shows a simplified scenario including a part with a flat surface but variable thickness (indicated by magenta color), which leads to a variable excitation angle. Even with constant fiber orientation assumed, this would lead to a change of the required transducer orientation along the part. Furthermore a surface with variable curvature would also make a dynamic positioning of the transducers essential.

In order to automate the process, two major components are therefore necessary:

\footnotetext{
${ }^{1}$ https://www.dlr.de/bt/en/desktopdefault.aspx/ tabid-2478/11208_read-53373/
} 


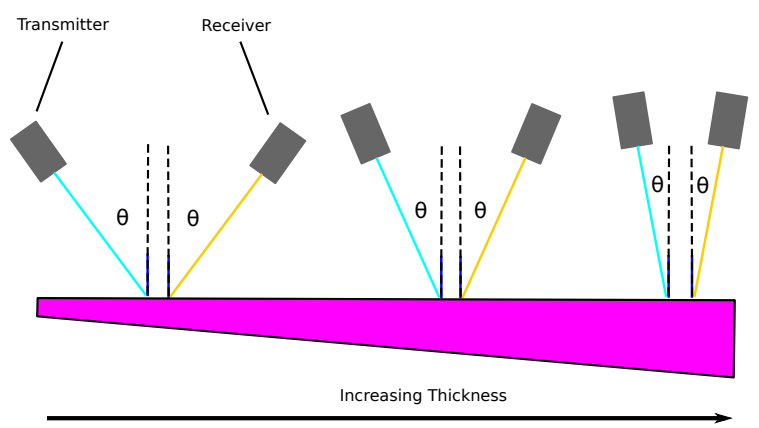

Figure 2: Change of excitation angle.

- A kinematic system that allows dynamic positioning of the transducers (an adaptive end-effector in our case)

- A software solution that, given a description of a part, creates a program for the kinematic system including the correct poses for the transducers

\section{SPECIMEN}

To validate the AEE, a testing specimen was designed. The following requirements were defined:

- The specimen should mimic real components and material used in aircraft.

- It must have all possible features that cause a change in the excitation angle, namely variable thicknesses (layup) and curvature.

- Artificial flaws must be inserted into the layup to enable the quantification of the probability of detection (POD) of the AEE

The specimen was manufactured from SAERTEX ${ }^{\circledR} 7006919$ dry fabric, infiltrated with RIMR135 resin by vacuum assisted resin infusion. The fiber volume content is $55 \%$. A schematic of the layup is drawn in Figure 3. Figure 3(a) shows a cross section through the laminate. The base laminate is a $2.2 \mathrm{~mm}$ thick, quasi-isotropic layup [0/90/-45/45] s. A step-like reinforcement layup $[0 / 90]_{4 \mathrm{~s}}$ was placed on top of the base laminate with a maximum thickness of $4.4 \mathrm{~mm}$. Therefore, the overall laminate thickness varies between 2.2 and $6.6 \mathrm{~mm}$. Artificial flaws made of $5 \times 5$ and $10 \times 10 \mathrm{~mm}^{2}$ square pieces of Kapton ${ }^{\mathrm{R}}$ sheet (DuPont Inc., Wilmington, DE, USA) were laminated into different depths. As shown in Fig. 4, the layup was performed in a small area close to the tail of an aircraft fuselage preform where significant curvature gradients occur. The specimen is $935 \mathrm{~mm}$ long, $505 \mathrm{~mm}$ wide, and has a maximum height of $110 \mathrm{~mm}$. The reinforcing layup covers a length of $420 \mathrm{~mm}$.

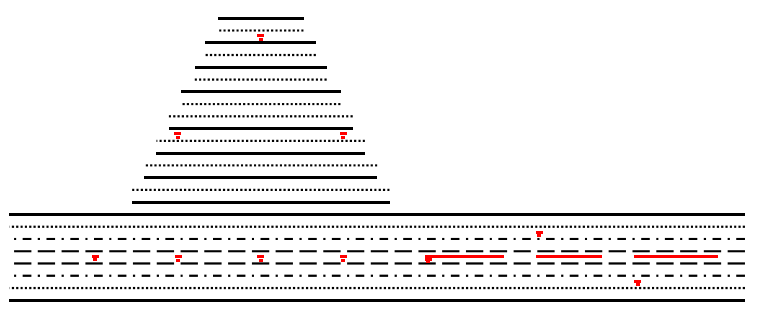

(a)

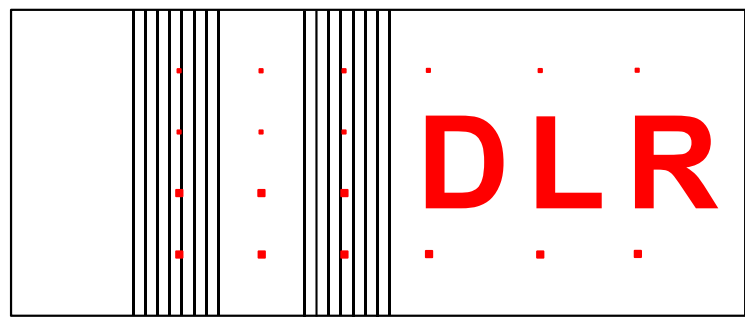

(b)

Figure 3: Cross sectional view (a) and top view (b) of the specimen's layup. Artificial flaws are drawn in red color. The dimensions are not true to scale.

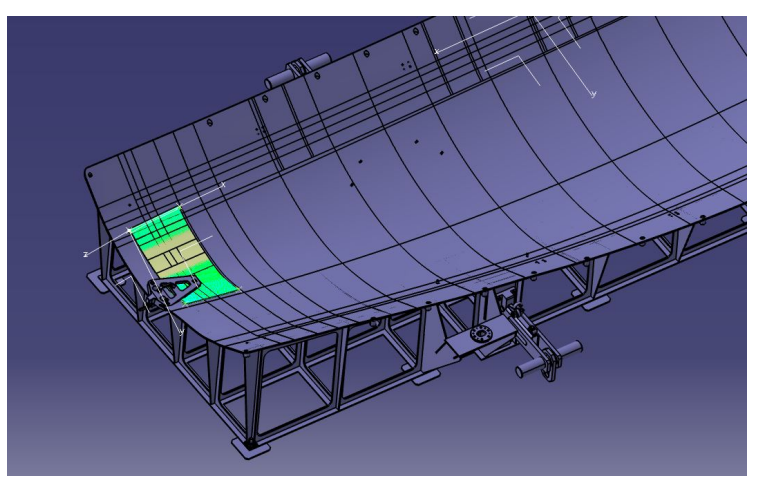

Figure 4: The layup of the specimen was done in an aircraft fuselage preform.

\section{SOFTWARE TOOLCHAIN}

It turned out that relying on commercial software was not sufficient for programming and controlling a system like the AEE, so a software toolchain had to be developed that provides the missing functionality. This section discusses our requirements, the shortcomings of commercial software in this respect, and our solution of the problem.

\subsection{Requirements}

To obtain a uniform scan of the part, a grid of $i$ measurement points on the specimen surface with the coordinates $x_{i}, y_{i}, z_{i}$ in the base coordinate system needs to be generated. This must be done based on a digital 
representation of the specimen. These are then used to create a program for the robotic system. To do so, they are put in order in such a way that the robot scans the specimen on a meander-like path. To fully define the kinematic path for the end-effector, the pose for both the transmitter and the receiver for each measurement point needs to be determined. Because the

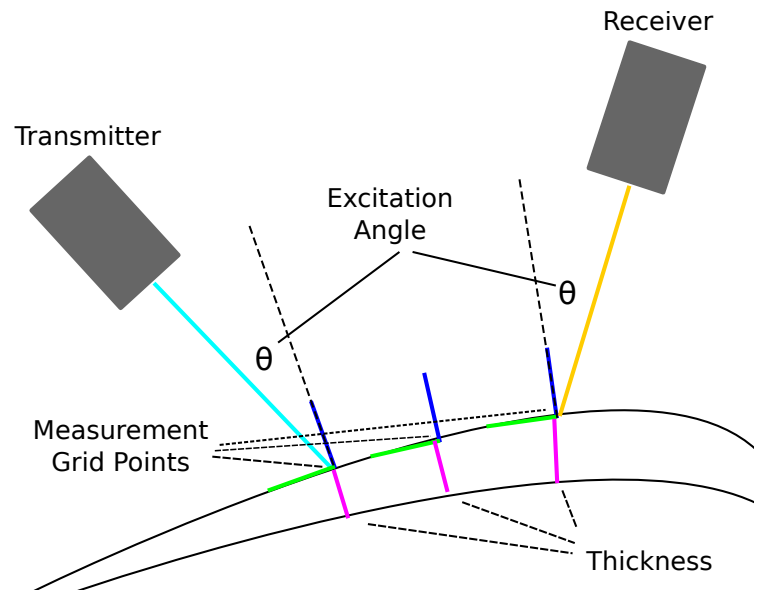

Figure 5: Determination of transmitter and receiver poses.

transducers should not collide with each other or the specimen, they need to be offset from each other and the surface. Also, the Lamb wave needs a minimum length it travels through the part. To determine the orientation of the ultrasonic heads the excitation angles $\theta_{i}$ need to be calculated. To enable this, the layup at each measurement point must be obtained, but this in turn requires the laminate thicknesses $d_{i}$. The distances which the ultrasound propagates in air and in the laminate also must always kept constant. The result is a list of transmitter-receiver pairs on the grid, depicted in figure 5.

\subsection{State-of-the-Art}

Usually computer-aided design (CAD) and offline programming (OLP) tools like CATIA $^{\text {TM }}$, DELMIA $^{\text {TM }}$, and FASTSURF ${ }^{\mathrm{TM}}$ (Dassault Systèmes, Vélizy-Villacoublay, France) are used for the path planning of robots in an industrial context. Here, one has a digital representation of the robotic environment, and the path as well as orientation of the endeffector can be generated based on the specimen's surface. However, the required additional kinematics of the AEE as well as the algorithm-driven variability of the transducer poses cannot be modeled neither programmed with these products in a sufficient way. Furthermore, the possibilities to generate the measurement grid on the specimen as well as the layup and thickness determintation $d_{i}$ are insufficient

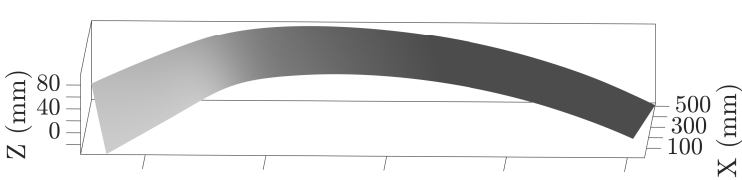

(a)

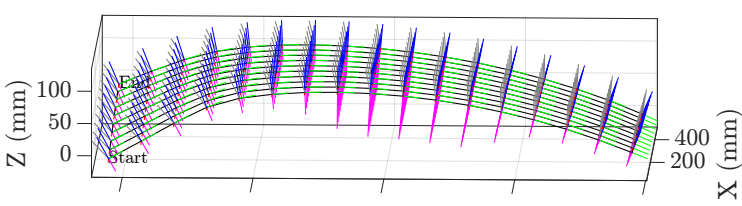

(b)

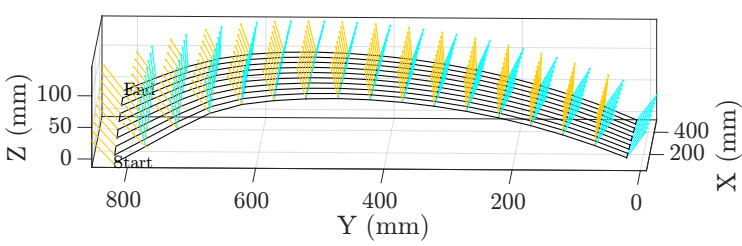

(c)

Figure 6: Offline programming in MATLAB ${ }^{\circledR}$. The STL file is loaded and fitted (a). The path, tripods, and excitation angles are generated (b). Then, the positions and orientations of the ultrasonic transducers are determined (c). In this example, a $50 \mathrm{~mm}$ grid is generated whereas a spacing of $2 \mathrm{~mm}$ is more realistic in a real measurement.

\section{in CATIA ${ }^{\mathrm{TM}}$.}

For the calculation of $\theta_{i}$, we purchased DISPERSE $^{2}$ (Imperial College London, London, UK). DISPERSE has been developed since the early $1990 \mathrm{~s}$ by Lowe and Pavlakovic, and was used for the validation of DC. DISPERSE is the leading software in its field and has some features not included in DC. However, it has a significant price while only available as a node-locked license and currently cannot calculate laminates containing more than 64 layers. DISPERSE also lacks an API, which renders us unable to automate the calculation of $\theta_{i}$ as we potentially have to calculate high numbers of sample points. For instance, a square grid with $2 \mathrm{~mm}$ spacings on the specimen has more then $10^{5}$ points. In DISPERSE you must enter each layup manually, calculate the complete dispersion diagram, and then extract the excitation angle of $\mathrm{A}_{0}$ at the transducer's frequency. These drawbacks have been one of the reasons why DC was created.

\subsection{Solution}

The four different software solutions mentioned in Section 4.2 which had been considered for the soft-

\footnotetext{
${ }^{2} \mathrm{http} / / /$ www.imperial.ac.uk/non-destructive-evaluation/ products-and-services/disperse
} 
ware tool chain initially were found insufficient later. Therefore, one task was to overcome their deficiencies and solve them by own implementation. Our tool chain uses two software componets currently, namely CATIA $^{\text {TM }}$ and MATLAB ${ }^{\circledR}$.

The basis is the CAD model of the specimen in CATIA $^{\mathrm{TM}}$. A separate tesselation of the top and bottom surfaces of the specimen is performed and the result is exported into two STL files. These files can be loaded into MATLAB ${ }^{\circledR}$. The top surface of the specimen is shown in Fig. 6(a). The vertices of the top surface are used as an input for the built-in MATLAB ${ }^{\circledR}$ Curve Fitting app (it requires the Curve Fitting Toolbox) to obtain a three-dimensional fit. Then the spacing of the measurement grid is defined and whether the transducers should move in lines parallel to the $x$ axis or $y$ axis (called "sweep direction" below). Square grid coordinates $x_{i}, y_{i}$ are generated in the $x-y$ plane, covering the $x-y$ range of the point cloud of the top surface. The grid coordinates are ordered in such a manner that alternating sweeps in the positive and negative sweep direction are performed. By evaluating the fitting function at $x_{i}, y_{i}$, the yet missing third coordinates $z_{i}$ can be obtained. The resulting path is indicated by the black lines in Figs. 6(b) and $6(\mathrm{c})$. At each measurement grid point $x_{i}, y_{i}, z_{i}$, the tripods $\vec{n}_{i}, \vec{b}_{i}, \vec{t}_{i}$ can now be calculated indicated by, respectively, blue, red, and green lines in Fig. 6(b).

Next, the thicknesses $d_{i}$ are determined by probing. The algorithm uses the surface normals $\vec{n}_{i}$, seeks the vertex of the lower surface, which is pierced by $\vec{n}_{i}$, and calculates the normal distance $d_{i}$ between this vertex and the corresponding grid point. The thicknesses $d_{i}$ are indicated by magenta colored lines in Fig. 6(b) (their lengths are scaled up by a factor of ten for clarity). With the now known $d_{i}$, the local layup can be obtained as follows. It is known that the specimen has twenty-four layers where it is $6.6 \mathrm{~mm}$ thick. Hence, the number of layers at any given measurement point from $d_{i}$ can be deduced. Then, from the sequence of layer orientations in the "maximum" layup, the local layup at the $i$ th measurement point can be obtained.

For the $\theta_{i}$ calculation, the basic algorithm used by DC was used. Therein, depending on the sweep direction, the propagation of Lamb waves is supposed to be either along the 0 or $90^{\circ}$ direction of the layup. Instead of complete dispersion diagrams $\theta_{i}(f)$, where $f$ covers a broad frequency range, only $\theta_{i}(200 \mathrm{kHz})$ for the $\mathrm{A}_{0}$ Lamb wave are calculated.

The excitation angles $\theta_{i}(200 \mathrm{kHz})$ vary between $14.4^{\circ}$ where the specimen is $2.2 \mathrm{~mm}$ thick and $13.2^{\circ}$ where it is $6.6 \mathrm{~mm}$ thick. The excitation angles are indicated by gray lines in Figure 6(b).
Now that that the coordinate system frames and excitation angles for each measurement point are known, the transmitter and receiver poses can be calculated. This is done by applying a local coordinate system displacement that adjusts the poses of the ultrasonic heads according to the excitation angle and fixed distance offsets on and from the surface (see Figure 5 ). Throughout the experiments discussed in this paper, $50 \mathrm{~mm}$ for both offsets are used. The final prepared data set is called the "excitation angle map" (EAM).

\section{ROBOTIC SETUP}

The process requires that the position of the transmitter and the receiver with respect to each other varies over time according to the EAM. That is why a single kinematic is not sufficient to implement the process. At the DLR site in Augsburg there are a number of industrial-grade robot cells available. The idea to implement the process was to use one of the industrial robots and attach a second smaller kinematic arm. The industrial robot serves as a kinematic for

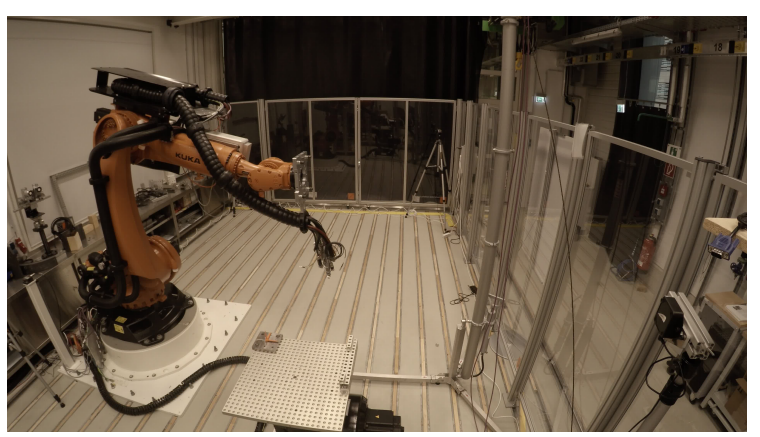

Figure 7: Robot cell.

positioning the transmitter, while the second attached arm is responsible for positioning the receiver. The robotic cell that served as a basis for evaluation of the adaptive testing is shown in figure 7 . The robot is a KUKA KR120 2700 HA run by a KRC4 controller. ${ }^{3}$

\subsection{End-effector Design}

First preliminary tests have been conducted with a KUKA LBR iiwa as a second kinematic attached to the KR120, as this robot arm was available at the DLR site in Augsburg already. 4 These tests have been promising, that is why a dedicated end-effector was

\footnotetext{
${ }^{3}$ https://www.kuka.com/en-de/products/robotsystems/industrial-robots/kr-quantec

${ }^{4}$ https://www.kuka.com/en-de/products/robotsystems/industrial-robots/lbr-iiwa
} 
designed. The new design should be as compact and as simple as possible, so it was decided to reduce the functionality to the necessary minimum (see figure 8).

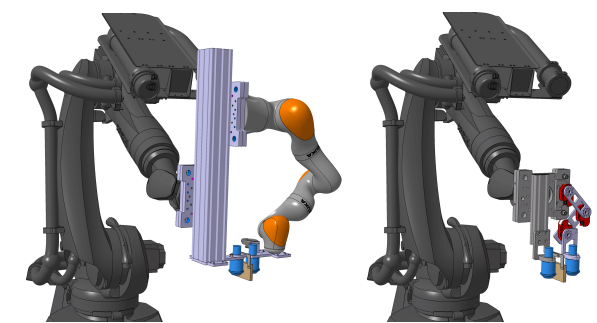

Figure 8: End-effector with iiwa (left) and new end-effector.

It was found sufficient that the ultrasonic transducers can move in a planar way to each other with additional rotation, so at least three degrees of freedom are necessary. Basically this can be implemented as e.g. a combination of two prismatic joints and one revolute joint or three coplanar revolute joints. The latter has been chosen because it was assessed that this way it is easier to build a compact, collision-free design with the neccessary workspace. We had been provided with three X-Series actuators by $\mathrm{HEBI}^{5}$ Robotics $^{\mathrm{TM}}$.

The end effector consists of a quick-release plate which is a standard at the DLR Augsburg, an aluminum profile and the three X-Series actuators. The actuators were mounted on aluminum links as close as possible to each other without collision. The default position of the static and the moving ultrasonic transducers are parallel to each other and the two links in a 45-90-45 degree position. With this default position, a widely adjustable angle between the transducers is possible. One advantage of this configuration is reducing the torque on axis 1 and 2 while providing a good workspace. An option to mount a direct sound shield (like the cardboard shield shown in figure 1) was added. Figure 9 shows the design.
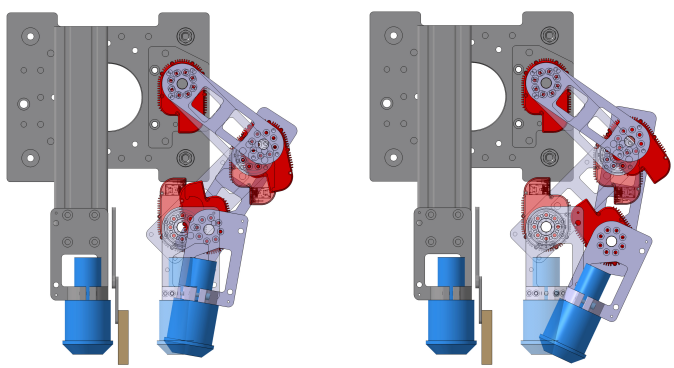

Figure 9: Re-designed end effector.

To increase the precision of the end-effector, all of the fixed parts as well as the quick release plate of the end effector were equipped with counterbores that can be measured with a $\mathrm{LEICA}^{\mathrm{TM}}$ laser tracker

\footnotetext{
${ }^{5}$ https://www.hebirobotics.com/
}

that is available at the DLR Augsburg site. This way the intrinsic dimensions of the end-effector could be calibrated with high precision, merely leaving the position accuracy of the actuators as a source of imprecision.

Four markers that help to calibrate the part in the robot cell have been addded to the corners of the specimen part described in section 3. To position the specimen part for the process, a framework to mount the specimen on had to be manufactured. The framework was derived in CATIA from the lower face of the part and produced on a RIDDER ${ }^{6}$ WariCut waterjet cutter. This was also used on the waterjet cutter for trimming the specimen. With this form fitting framework, it is easy to place the specimen precisely on a welding table in front of the robot repeatable and so it is not necessary to recalibrate its position after removing it. The framework and the specimen are shown in figure 10.

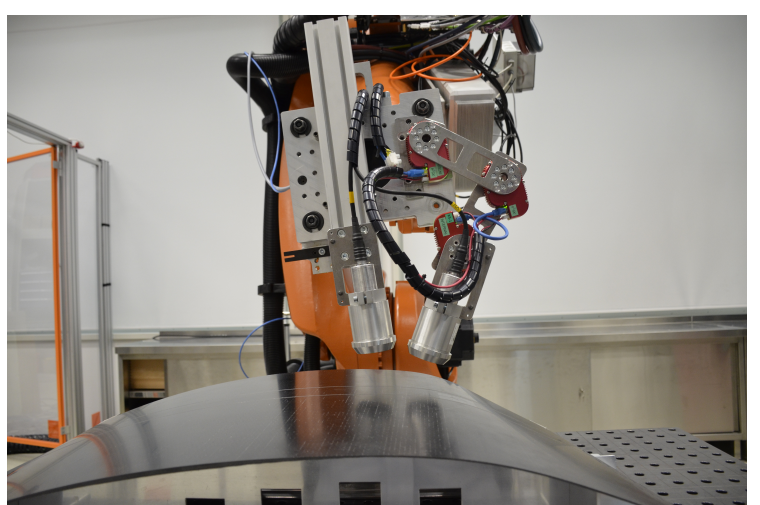

Figure 10: Specimen mounted on the framework and assembled end-effector (direct sound shield not mounted).

\subsection{Process Control}

To make the process work, two main aspects had to be taken into consideration:

- The KUKA robot and the HEBI arm have to follow the trajectory defined by the transmitter / receiver poses as waypoints

- The trajectories need to be synchronized in a way so that both robots reach each waypoint at the same time

For the following experiments, a master-slaveprinciple was implemented: The transmitter is mounted on the KUKA robot that moves along the specimen. The receiver is mounted on the HEBI arm, which adjusts itself according to the position of the transmitter. In order to generate a trajectory for the KUKA robot, a KRL (KUKA Robot Language)

\footnotetext{
${ }^{6} \mathrm{https}: / /$ www.ridder.de/
} 
robot program for its robot controller gets generated from the calculated transmitter poses. This is done by a KRL generator that was implemented as part of our MATLAB toolchain discussed earlier (Section 4). The HEBI robotics API includes a generic kinematics solver, but for our purpose a forward and inverse kinematic solver was implemented specifically for our arm configuration. The poses for the HEBI arm also get generated from MATLAB as a $\mathrm{CSV}^{7}$ file.

The robot controller sends the transmitter pose and the current waypoint number to a PC in realtime over the KUKA robot sensor interface (RSI) in a $4 \mathrm{~ms}$ $(\equiv 250 \mathrm{~Hz}$ ) cycle. The PC uses the transmitter pose and the waypoint number to interpolate the receiver pose in realtime and uses the HEBI API to replan the arm trajetory in realtime accordingly. The feedback of the HEBI arm runs at a $5 \mathrm{~ms}(\equiv 200 \mathrm{~Hz})$ cycle.

\section{EXPERIMENTAL EVALUATION}

As a first step, a set of parameters for the ultrasound device was determined experimentally. This was done by moving the end-effector across the part with manual control and adjusting the settings of the ultrasound device so that the measured signal was reasonable in terms of amplitude, phase and other factors. Once a good set of parameters was found, it was kept constant for all the following tests. Instead of cardboard, a piece of EPDM ${ }^{8}$ rubber was used as a direct sound shield.

Throughout all the tests, the trajectory of the KUKA robot was generated from the calculated transmitter poses, i.e. the pose of the transmitter can be assumed to be correct all the time. The speed of the KUKA was set to $100 \mathrm{~mm} / \mathrm{s}$. Then the following tests have been done.

1. Keep the receiver pose in a configuration that is correct for the thick part in the middle of the specimen, scan the whole part with the receiver in this configuration

2. Keep the receiver pose in a configuration that is correct for the area with the strongest curvature, scan the whole part with the receiver in this configuration

3. Scan the whole part using the adaptive process, with the receiver adjusting its pose continuously

Test 1 and 2 basically mimic a non-adaptive endeffector (like the conventional end-effector shown in figure 1) with the transmitter and receiver being in a fixed configuration to each other.

\footnotetext{
${ }^{7}$ comma separated value

8 ethylene propylene diene monomer
}

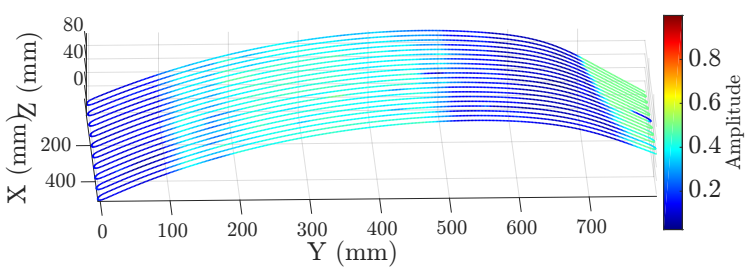

Figure 11: Result with receiver pose adjusted to thickest area.

Figure 11 shows the result of test 1 . As expected, a reasonable amplitude can mainly be observed in the thick area of the part. The amplitude is not at a maximum, which is because the power settings initially had to be set to a lower level that does not generate overdrive in the thinner areas.

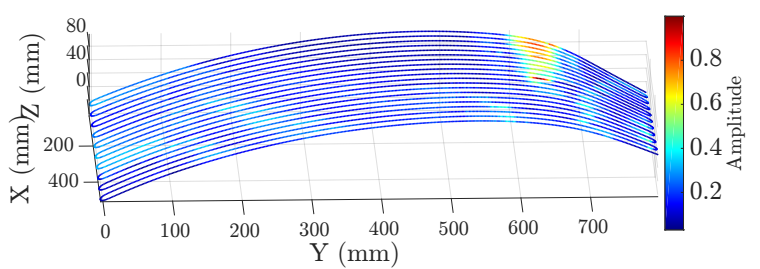

Figure 12: Result with receiver pose adjusted to strongest curvature.

The result of test 2 is depicted in figure 12. One can see that a significant amplitude gets detected in the strongly curved area. But because the pose of the receiver is incorrect for other areas of the specimen, no usable signal can be observed there.

The result of test 3 , using the adaptive process, is shown in figure 13. One can see that generally a signal can be obtained throughout the whole surface area. A less strong amplitude can be observed in the thicker area, which is expected. This result confirms

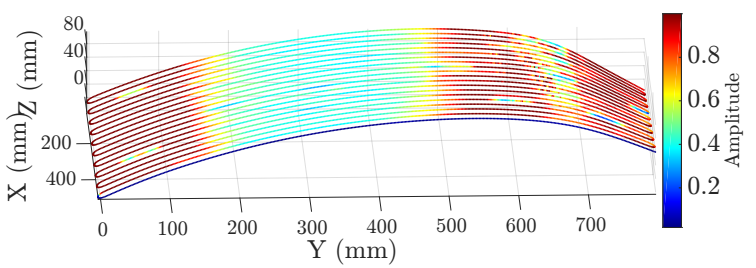

Figure 13: Result of the adaptive process.

that the adaptive process generally is working according to our assumptions.

However, there is still a lot of aspects to investigate and a lot of room for improvement. For instance, the precision is still quite coarse and the flaw detection not sufficiently reliable. Some flaws show up in the scan (see figure 13), some do not. Also the influence of varying the process parameters has not been investigated in detail. Multiple factors for improvement have been identified: 
Direct Sound Shield: The shield built into the endeffector is mounted in a fixed position. In some areas of the part, a little gap between the surface and the part could be observed, allowing sound directly travelling from the transmitter to the receiver, influencing the lamb wave amplitude detection.

Lamb Wave Propagation Length: Fixed lengths of $50 \mathrm{~mm}$ for the offsets on and from the surface were used. While the distance from the surface could be reduced, the transducers cannot be moved more closely together because of their size. This causes the flaws to appear "stretched" along the sweep direction.

Process Control Accuracy: So far, no in-depth tests have been conducted how accurate the process control system works, i.e. how precise the transducers follow the waypoints and how well their trajectories are synchronized.

\section{CONCLUSIONS AND FUTURE WORK}

In this paper, we have introduced an approach for aircoupled ultrasonic inspection through adaptive lamb wave control. A software toolchain to fully calculate and determine the adaptive process was developed. An adaptive end-effector was designed for experimental evaluation. For this purpose, a specimen part was manufactured.

It was possible to verify the principle and the toolchain as well as the adaptive end-effector were successfully tested. While the process was found to be working generally, still a lot of room for improvement exists. Future work could include improving the hardware such as the direct sound shield. Furthermore, diffent combinations of ultrasonic heads and other specimens could be examined. Also, a sophisticated analysis of the process control system could be revealing.

\section{ACKNOWLEDGEMENTS}

We would like to thank HEBI Robotics ${ }^{9}$ for providing us a set of X-Series Actuators for the experimental evaluation.

\section{REFERENCES}

Castaings, M. and Cawley, P. (1996). The generation, propagation, and detection of Lamb waves in plates us-

\footnotetext{
${ }^{9}$ https://www.hebirobotics.com/
}

ing air-coupled ultrasonic transducers. J. Acoust. Soc. Am., 100(5):3070-3077.

Castaings, M., Cawley, P., Farlow, R., and Hayward, G. (1998). Single sided inspection of composite materials using air coupled ultrasound. J. Nondestr. Eval., 17(1):37-45.

Castaings, M. and Hosten, B. (2001). Lamb and SH waves generated and detected by air-coupled ultrasonic transducers in composite material plates. NDT\&E Int., 34:249-258.

Castaings, M. and Hosten, B. (2008). Ultrasonic guided waves for health monitoring of high-pressure composite tanks. NDT\&E Int., 41:648-655.

Cunfu, H., Hongye, L., Zenghua, L., and Bin, W. (2013). The propagation of coupled Lamb waves in multilayered arbitrary anisotropic composite laminates. $J$. Sound Vib., 332:7243-7256.

Diamanti, K. and Soutis, C. (2010). Structural health monitoring techniques for aircraft composite structures. Prog. Aeronaut. Sci., 46:342-352.

Huber, A. M. A. and Sause, G. R. (2018). Classification of solutions for guided waves in anisotropic composites with large numbers of layers. J. Acoust. Soc. Am., 144(6):3236-3251.

Kessler, S. S., Spearing, S. M., and Soutis, C. (2002). Damage detection in composite materials using Lamb wave methods. Smart Mater. Struct., 11:269-278.

Kundu, T., Maji, A., Gosh, T., and Maslov, K. (1998). Detection of kissing bonds by Lamb waves. Ultrasonics, 35:573-580.

Lowe, M. J. S., Alleyne, D. N., and Cawley, P. (1998). Defect detection in pipes using guided waves. Ultrasonics, 36:147-154.

Lowe, M. J. S. and Cawley, P. (1994). The applicability of plate wave techniques for the inspection of adhesive and diffusion bonded joints. J. Nondestr. Eval., 13(4):185-200.

Luukkala, M., Heikkila, P., and Surakka, J. (1971). Plate wave resonance - A contactless test method. Ultrasonics, 9(4):201-208.

Luukkala, M. and Meriläinen, P. (1973). Metal plate testing using airborne ultrasound. Ultrasonics, 11(5):218221.

Maslov, K. and Kundu, T. (1997). Selection of Lamb modes for detecting internal defects in composite laminates. Ultrasonics, 35:141-150.

Purekar, A. S. and Pines, D. J. (2010). Damage detection in thin composite laminates using piezoelectric phased sensor arrays and guided Lamb wave interrogation. $J$. Intell. Mater. Syst. Struct., 21:995-1010.

Ramadas, C., Balasubramaniam, K., Joshi, M., and Krishnamurthy, C. V. (2011). Sizing of interface delamination in a composite T-joint using time-of-flight of Lamb waves. J. Intell. Mater. Syst. Struct., 22:757768.

Solodov, I., Pfleiderer, K., Gerhard, H., and Busse, G. (2004a). Nonlinear acoustic approach to material characterisation of polymers and composites in tensile tests. Ultrasonics, 42:1011-1015. 
Solodov, I., Pfleiderer, K., Gerhard, H., Predak, S., and Busse, G. (2006). New opportunities for NDE with air-coupled ultrasound. NDT\&E Int., 39(3):176-183.

Solodov, I., Stoessel, R., and Busse, G. (2004b). Material characterization and NDE using focused slanted transmission mode of air-coupled ultrasound. Res. Nondestr. Eval., 15(2):65-85.

Su, Z., Ye, L., and Lu, Y. (2006). Guided Lamb waves for identification of damage in composite structures: A review. J. Sound Vib., 295:753-780.

Toyama, N., Noda, J., and Okabe, T. (2003). Quantitative damage detection in cross-ply laminates using Lamb wave method. Compos. Sci. Technol., 63:1473-1479.

Ullmann, T., Shi, Y., Rahner, N., Schmücker, M., Fey, P., Busse, G., and Becker, S. (2012). Quality Assurance for the Manufacturing of Oxide Fiber Reinforced Ceramic Composites for Aerospace Applications. In 4th International Symposium on NDT in Aerospace, pages $1-11$.

Viktorov, I. (1967). Rayleigh and Lamb Waves: Physical Theory and Applications. Plenum, New York.

Wilcox, P. D., Lowe, M. J. S., and Cawley, P. (2001). Mode and transducer selection for long range Lamb wave inspection. J. Intell. Mater. Syst. Struct., 12:553-565. 